\title{
Acute effect of moderate intensity physical exercise on postprandial hyperlipaemia of individuals with central obesity ${ }^{*}$
}

\author{
Djeyne Silveira Wagmacker ${ }^{1,2}$, Karla Kurzendorff Souza², Julia Vieira da Graça ${ }^{2}$, \\ Geodânia da Costa Barreto², Paula Caroline Souza Macêdo², Tâmara Felix Ferreira², \\ Ana M. Ladeia ${ }^{1 \#}$ \\ ${ }^{1}$ Bahian School of Medicine and Public Health, Science Development Foundation of Bahia, Bahia, Brazil; \\ \#Corresponding Author: analadeia@uol.com.br \\ ${ }^{2}$ College of Adventist Bahia, Bahia, Brazil
}

Received 5 November 2012; revised 7 December 2012; accepted 15 December 2012

\begin{abstract}
Introduction: High postprandial triglyceride levels and obesity are associated with coronaropathy, and influence the pathogenesis and progression of atherosclerosis. Objective: To evaluate the effect of immediate acute physical exercise on postprandial hyperlipaemia in individuals with central obesity. Methods: Study included sedentary, normolipidemic individuals aged 18 - 30 years, with waist circumference $>80$ in for women and $>94$ for men. The physical exercise was performed for 45 minutes with $55 \%$ $60 \%$ of the heart rate reserve. Triglyceride level tests were performed after fasting, and at time intervals of $1 \mathrm{~h}$ and $3 \mathrm{~h}$ after lipid overload, a week before and immediately after a session of physical exercise. Results: Thirty-six individuals were evaluated (mean age $23.74 \pm 4.05$ years, $88.2 \%$ women, $\mathrm{BMI}=28.4 \pm 3.4$ ). Fasting and postprandial triglyceride levels were similar before and after the exercise $(p=0.87)$. A smaller rise in triglyceride levels was observed in the 1st hour in obese individuals (5\%) compared with overweight individuals $(21 \%) p=0.001$. Conclusion: In individuals with central obesity, moderate intensity physical exercise performed immediately before lipid overload does not reduce the hyperlipidemic peak.
\end{abstract}

Keywords: Postprandial Hyperlipaemia; Exercise; Central Obesity

\section{INTRODUCTION}

The relationship between lipid levels and the devel-

"Financial support: This study had no external funding sources. opment of atherosclerotic diseases has been studied for many decades [1]. It is known that elevated levels of fasting plasma triglycerides is associated with a $14 \%$ increase in the risk of coronary artery disease for men and a $37 \%$ increase for women [2].

The postprandial lipaemia is an event that always occurs after a meal containing lipids, characterized as transitional hipertriglyceride state and it is associated with varied metabolic events such as changes in pro-atherogenic and prothrombotic factors. Growing evidence has supported the hypothesis that high concentratios of triglycerides in plasma on the postprandial period are more rugged predictors of coronary artery disease by influencing its pathogenesis and progression [3].

Western populations present a standard diet rich in fat (50 - $70 \mathrm{~g} / \mathrm{meal}$ ) [4] and spend most of the day in the postprandial state $(14-18 \mathrm{~h})[5,6]$, however, the usual lipid profile research protocols do not include the evaluation of postprandial lipaemia.

The accumulation of abdominal fat, denominated central, visceral or androgenic obesity, has been strongly associated with the development of atherosclerotic disease. Obesity influences the increase in insulin resistance, which leads to an increase in very low density lipoprotein (VLDL) production by the liver, influencing the development of a state of hypertriglyceridemia and exaggerating the state of postprandial hiperlipidemia [7].

Although the effects of physical exercise on attenuating the triglyceride levels are known, the literature still remains controversial with regard to the immediate acute effects. Most studies evaluate the late acute effect $12 \mathrm{~h}$ post-exercise, and the influence of this time on the lipid curve. Studies that have evaluated the immediate acute response diverge with respect to the exercise protocol used, present reduced sample size, and included nonobese individuals [7-13]. Furthermore, these studies have 
also presented contradictory results about the lipid curve: in some studies [3-8] the exercise it was shown to have a reducing effect and on others [9-12] no effect was evidenced. Therefore, the aim of this study was to evaluate the effect of acute moderate intensity physical exercise on the lipid response in patients with central obesity by means of a self controlled intervention study.

\section{METHODS}

\subsection{Population}

The research was conducted at the School Clinic of a higher education institution, from September 2011 to May 2012. All the individuals registered in the service, who presented central type obesity were invited to participate in the study. Forty volunteers met the following inclusion criteria of the study: age between 18 to 30 years, waist circumference $>80 \mathrm{~cm}$ for women and $>94$ $\mathrm{cm}$ for men [12] and sedentarism, the latter determined by the International Physical Activity Questionnaire Short version (IPAQ) [14]. This questionare consists of eight discursive questions that provide information about the duration (minutes/day) and frequency (days/week) of trekking and every day activities performed in sitting position or that require moderate and vigorous physical effort, classifying the level of physical activity into 4 categories (sedentary, insufficiently active, active and very active [15]. The exclusion criteria were: individuals who presented cardiovascular disease, musculoskeletal, metabolic or other pathology that would limit performance of the physical activity, history of alcoholism, use of lipidlowering drugs, corticoids, diuretics, beta-blockers, contraceptives, hypothyroidism, renal parenchymal disease or diabetes. After the initial evaluation 5 individuals were excluded because they had dyslipidemia.

A sample size of 32 pairs was calculated to obtain a statistical power of $80 \%$ in the detection of differences of $10 \mathrm{mg} / \mathrm{dl}$ in the Triglyceride (TG) values, with an alpha of $5 \%$ considering a standard deviation of $20 \mathrm{mg} / \mathrm{dl} \mathrm{[16].}$ The sample was extended to include 34 individuals.

\subsection{Study Design}

This was an analytical, prospective intervention study. The patients included in the study went through three stages of evaluation: in stage 1 , the baseline evaluation was made; in stage 2 the lipid overload was performed, standardized as the control day, and in stage 3 the physiccal exercise was performed, followed by lipid overload serving as the experiment day.

\subsection{Baseline Evaluation or Initial}

The selected volunteers answered a standard questionnaire and were subjected to the physical exam. The questionnaire contained self reported information about age, sex, ethnicity, educational level. The physical exam consisted of cardiac frequency, arterial pressure at rest, total body mass, stature, waist and hip circumference measurements. The waist was measured with a metric tape at the point defined by half the distance that separates the iliac crest from the last rib; the hip was measured at the point of largest circumference in the gluteal region. The weight and height measurements were used to calculate the body mass index by the formula: weight/height ${ }^{2}$. The waist-hip ratio (WHR) was calculated by dividing the waist measurement value by that of the hip [17].

All individuals underwent a resting electrocardiogram (ECG) exam, performed by means of a computerized electrocardiogram (ErgoPc-version 5.0), with the recording of the 12 standard leads. In addition, they performed the ergometric test on a treadmill, using the ramp protocol. Tests were interrupted by intense physical tiredness. During the entire ET, the individual was monitored by ECG, and blood pressure was measured by the auscultatory method. The test was performed by a cardiologist.

\subsection{Control Day}

Seven days after the maximum physical effort test, the volunteers were subjected to a lipid overload test, after fasting for 12 hours. The individuals were previously instructed to abstain from alcohol and caffeine at dinner the night before the exam, in order to avoid additional lipid overload before and during the 24 hours that followed the lipid overload test.

Blood was collected at three time points: baseline (T0), 1 hour after lipid overload (T1) and 3 hours after lipid overload (T3). At the baseline collection, TG, Total cholesterol (TC), intermediate density lipoprotein (HDL) and glucose were dosed. The Low Density Lipoprotein (LDL) evaluation was calculated by the Friedwald formula [13]. After the baseline blood collection, lipid overload was induced in subjects, by the ingestion of an industrialized liquid lipid compound, marketed by TECNOVIDA, containing 50 grams of triglycerides and 4.3 grams of carbohydrates. The compound was ingested in a maximum time of 5 minutes. After the ingestion of fat, blood samples were collected within time intervals of 1 and $3 \mathrm{~h}$, to measure triglycerides. The subject remained at rest, in the sitting position, in the laboratory environment during the entire postprandial period of $3 \mathrm{~h}$, getting up only when they needed to use the bathroom.

For the TG analysis, $5 \mathrm{ml}$ of blood were collected in tubes with EDTA (anticoagulant and chelating agent), and after collection, the blood samples were centrifuged at a speed of 3400 rotations/min for 5 minutes. The TGs were evaluated by the enzymatic method using kits available commercially and analysed on an Advia 120 
device (Bayer, Alemanha).

\subsection{Exercise Day}

Seven days after the first lipid overload a second lipid overload was induced, proceeded by physical exercise. After the baseline blood collection, the individuals were subjected to a continuous exercise on an ergometric treadmill, for 45 minutes. The the first seven minutes of exercise was performed at low intensity ( $40 \%$ of maximum $\mathrm{CF}$ ), a phase regarded as the warm up; 35 minutes at moderate intensity ( $60 \%$ of maximum CF), the phase corresponding to the training itself and 3 minutes of slowdown at decreasing intensity.

The training cardiac frequency was calculated by the Karvonen formula, $[18,19]$ using the maximum CF achieved during the ergometric test.

During the test, the arterial pressure, cardiac frequency and subjective perception of the participants' effort were monitored. Immediately after the physical exercise, the lipid overload was induced and blood samples were collected at the time intervals of 1 and $3 \mathrm{~h}$.

\subsection{Ethical Aspects}

The study was submitted to the Research Ethics Committee of the "Faculdade Adventista da Bahia". The administration of fat in the study $(50 \mathrm{~g})$ did not hurt ethical criteria since the Western diet, on an average contains 20 $70 \mathrm{~g}$ fat per meal [4]. After the intervention protocol of this study had been performed, all the participants in the research were referred to the cardiology sector for follow-up by one of the participating institution, where they were offered a free, supervised physical activity program for 3 months.

\subsection{Statistical Analysis}

For comparison of plasma TG over time with and without physical exercise, the ANOVA test for repeated measures was applied. The absolute and percentage differences of TG between the baseline and time interval 3 hours after lipid overload were compared by the Student's- $t$ test for paired samples. The data were previously analyzed for symmetry by the Kolmogorov-Smirnov test. All data analyses were performed using the software SPSS (Statistical Package for Social Science) version 14.0. The results were expressed as average \pm standard deviation. The levels of significance were established at $5 \%$.

\section{RESULTS}

The population was composed of 36 young, adult individuals, with a mean age of $23.5 \pm 3.8$ years, with predominance of females $(87.5 \%)$. The mean BMI was 28.4 $\pm 3.4 \mathrm{~kg} / \mathrm{m}^{2}$; waist circumference $(\mathrm{WC})=95.5 \pm 0.7 \mathrm{~cm}$ and $89.8 \pm 6.6 \mathrm{~cm}$ for males and females respectively. All clinical characteristics are described in Table 1.

When the TG values were analyzed over the course of the 3 hours, a steep increase in the TG values was observed from the 1st hour for both the control day and exercise day, an no differences in the rise in triglycerides were identified between days with and without physical exercise until the third hour $(\mathrm{p}=0.87)$ (Figure 1).

When the rise in absolute and percentage values between fasting and other peaks of the curve, 1st and 3rd hour respectively, were compared, no differences were observed between the days with and without exercise. (Table 2).

On the day without exercise, when the $\Delta$ (1st hourfasting) absolute and percentage of the triglycerides were compared, a percentage rise of 5\% for the obese and $21 \%$

Table 1. Socio-demographic, clinical and anthropometric characteristics of individuals with central obesity $(n=36)$.

\begin{tabular}{|c|c|}
\hline VARIABLES & \\
\hline Age (years) & $23.74 \pm 4.05^{*}$ \\
\hline Gender & $\mathrm{n}(\%)$ \\
\hline Female & $30(88.9)$ \\
\hline Male & $4(11.1)$ \\
\hline \multicolumn{2}{|l|}{ Color } \\
\hline White & $13(36.1)$ \\
\hline Black & $5(13.9)$ \\
\hline Brown & $17(47.2)$ \\
\hline \multirow[t]{2}{*}{ Yellow } & $1(2.8)$ \\
\hline & Mean \pm SD \\
\hline \multicolumn{2}{|l|}{ Waist circumference $(\mathrm{cm})$} \\
\hline Men & $95.5 \pm 0.7$ \\
\hline Women & $89.8 \pm 6.6$ \\
\hline \multicolumn{2}{|l|}{ Waist hip ratio $(\mathrm{cm})$} \\
\hline Men & $0.93 \pm 0.05$ \\
\hline Women & $0.85 \pm 0.06$ \\
\hline Body mass index $\left(\mathrm{kg} / \mathrm{m}^{2}\right)$ & $28.4 \pm 3.4$ \\
\hline Triglycerides (mg/dl) & $92.9 \pm 34.9$ \\
\hline Total cholesterol (mg/dl) & $168.1 \pm 34.5$ \\
\hline Low density lipoprotein (mg/dl) & $105.8 \pm 31.3$ \\
\hline High density lipoprotein (mg/dl) & $42.7 \pm 7.2$ \\
\hline Glycemia (mg/dl) & $80.8 \pm 11.6$ \\
\hline Systolic arterial pressure (mmHg) & $113.3 \pm 7.4$ \\
\hline Diastolic arterial pressure $(\mathrm{mmHg})$ & $75.9 \pm 5.6$ \\
\hline
\end{tabular}




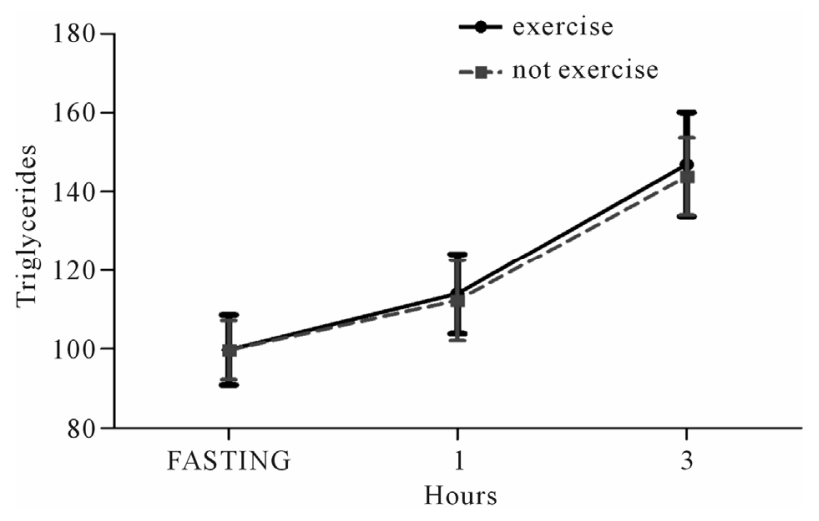

Figure 1. Analysis of repeated measures of the triglycerides.

Table 2. Comparison of the absolute and percentage difference of basal triglycerides and peaks of the lipid curve of the control and exercise day of individuals with central obesity $(n=36)$.

\begin{tabular}{lccc}
\hline & Without exercise & Exercise & $\mathrm{p}$ \\
\hline$\Delta$ absolute 1st hour (mg/dl) & $12.7 \pm 12.8$ & $12.8 \pm 20.3$ & 0.96 \\
$\Delta$ percentage 1st hour (mg/dl) & $13 \% \pm 15 \%$ & $11 \% \pm 17 \%$ & 0.46 \\
$\Delta$ absolute 3rd hour (mg/dl) & $42.2 \pm 36.2$ & $39.8 \pm 22.4$ & 0.72 \\
$\Delta$ percentage 3rd hour (mg/dl) & $49 \% \pm 35 \%$ & $46 \% \pm 28 \%$ & 0.61 \\
\hline
\end{tabular}

for the overweight ( $p=0.001)$ was identified, demonstrating a delay in the rise of triglycerides in the obese population. The difference in absolute values was $16.7 \pm 14.3$ for the overweight and $6.0 \pm 6.7$ for the obese $(p=0.006)$. No differences were found in the comparison of $\Delta$ (3rd hour-fasting) for the days with and without exercise (Table 3).

\section{DISCUSSION}

The late acute effects of physical exercise on postprandial lipaemia are well documented in the literature, and the best results have been shown when the exercise is performed up to $12 \mathrm{~h} \mathrm{[12]} \mathrm{before} \mathrm{the} \mathrm{lipid} \mathrm{overload,} \mathrm{how-}$ ever, the immediate acute effects on hyperlipaemia continue to be contradictory, and there are few studies that have used this methodology. In the present study acute exercise did not change the postprandial hyperlipaemia in individuals with central obesity in the time interval of $3 \mathrm{~h}$.

According to Katsanos [20] when the exercise is performed immediately before the ingestion of fat, the reduction in postprandial hyperlipaemia is attributed mainly to the lower production of VLDL by the liver, rather than the increase in lipase enzyme in the skeletal muscle. Considering that protein lipase activity is the main agent in reducing the lipid curve and its activity is only elevated 4 hours after exercise, according to Zahng, Thomas and Ball [12] the evaluation in 3 hours may not have been
Table 3. Comparison of $\Delta$ absolute and percentage of the triglycerides between the baseline period and the 1st hour of the individuals with central obesity and BMI greater than or less than $30 \mathrm{~kg} / \mathrm{m}^{2}(\mathrm{n}=36)$.

\begin{tabular}{|c|c|c|c|}
\hline & Overweight $(\mathrm{n}=25)$ & Obese $(n=11)$ & $\mathrm{p}$ \\
\hline \multicolumn{4}{|c|}{$\Delta$ absolute $1 \mathrm{st} \mathrm{h}(\mathrm{mg} / \mathrm{dl})$} \\
\hline Without exercise & $16.7 \pm 14.3$ & $6.0 \pm 6.7$ & 0.006 \\
\hline With exercise & $17 \pm 15.1$ & $13 \pm 13.3$ & 0.37 \\
\hline \multicolumn{4}{|c|}{$\Delta$ percentage 1 st h $(\%)$} \\
\hline Without exercise & $21 \% \pm 17 \%$ & $5 \% \pm 9 \%$ & 0.001 \\
\hline With exercise & $20 \% \pm 13 \%$ & $13 \% \pm 11 \%$ & 0.16 \\
\hline \multicolumn{4}{|c|}{$\Delta$ absolute $3 \mathrm{rd} \mathrm{h}(\mathrm{mg} / \mathrm{dl})$} \\
\hline Without exercise & $44.9 \pm 31.1$ & $44.4 \pm 54.3$ & 0.97 \\
\hline With exercise & $43.3 \pm 21.8$ & $42.8 \pm 25.6$ & 0.94 \\
\hline \multicolumn{4}{|c|}{$\Delta$ percentage $3 \mathrm{rd} \mathrm{h}(\%)$} \\
\hline Without exercise & $53 \% \pm 27 \%$ & $48 \% \pm 51 \%$ & 0.68 \\
\hline With exercise & $50 \% \pm 26 \%$ & $43 \% \pm 28 \%$ & 0.50 \\
\hline
\end{tabular}

been sufficient time in which to identify lipase activation.

Insulin resistance is another factor that may have an influence on attenuating the increase in TG in the postprandial period, however, this only begins to change $2 \mathrm{~h}$ after an acute session of exercise, according to the study of Zanhg et al. [7]. This finding is important to contribute to the reduction in hyperlipaemia, since insulin resistance and hyperinsulinemia promote hepatic VLDL-TG secretion and inhibits lipoprotein lipase activity, which has a negative influence on the clearance of lipoproteins rich in TG, thus increasing the hyperlipaemia in fasting and postprandial periods.

The results of this study are similar in methodology and results to that of Pfeiffer et al. [11] and Petridou et al. [21], who used moderate intensity exercise of long duration and also did not observe immediate acute effects on the reduction in postprandial hyperlipaemia.

The capacity to remove the TG after a meal rich in TG varies and may persist for up to $8 \mathrm{~h}$, with the peak being reached between the 3 rd and 4 th hour. The following are factors that interfere in the clearance of TG, and delay their removal from the bloodstream: the state of hyperinsulinemia, obesity, age, male sex, and sedentary lifestyle [7]. In the present study, it was observed that obese individuals had a slower rise in the 1st hour with increase in triglycerides of only $5 \%$ in comparison with baseline. This finding is in agreement with Blackburn et al. [22] who observed that the peak of hyperlipaemia in the obese occurs around the sixth hour.

On the other hand, on the exercise day no differences 
occurred between obese and overweight subjects/patients/ individuals, which may suggest a proximity of the lipid peak in comparison with overweight individuals, and it is possible that the influence of exercise is on the duration of the lipid curve and not on its magnitude, since no differences were perceived in the delta between the obese with and without exercise. However, the study presented limitations with respect to the time of observation of the lipid curve, since triglycerides were recorded only up to the peak of hyperlipemia and not during the entire curve.

Finally, this study contributed to confirmation of the hypothesis that exercise performed immediately before the lipid overload does not affect the peak of hyperlipaemia, which usually occurs between the 3rd and 4th hour after the ingestion of the lipid compound. On the other hand, the same cannot be concluded about the efficacy of physical exercise in the long term, since only the immediate acute effects were evaluated. For better definition of the efficacy and the magnitude of the late effects of the exercise in this population, further studies are needed, with longer follow-up of the lipid curve.

\section{REFERENCES}

[1] Dawber, T.R. (1980) The Framingham study: The epidemiologic of atherosclerotic disease. Harvard University Press, Cambridge, 14-29.

[2] Austin, M.A. Hokanson, J.E. and Edwards, K.L. (1998) Hypertriglyceridemia as a cardiovascular risk factor. American Journal of Cardiology, 81, 7B-12B. doi:10.1016/S0002-9149(98)00031-9

[3] Teixeira, M., et al. (2006) Efeitos do exercício agudo na lipemia pós-prandial em homens sedentários. Arquivos Brasileiros de Cardiologia, 87, 3-11. doi:10.1590/S0066-782X2006001400002

[4] Lairon, D. (1996) Nutritional and metabolic aspects of post prandial lipemia. Reproduction Nutrition Development, 36, 345-355. doi:10.1051/rnd:19960401

[5] Moreton, J.R. (1950) Chylomicronemia, fat tolerance and atherosclerosis. Journal of Laboratory and Clinical Medicine, 35, 373-384.

[6] Zilversmit, D.B. (1979) Atherogenesis: A postprandial phenomenon. Circulation, 60, 473-485. doi:10.1161/01.CIR.60.3.473

[7] Zhang, J.Q., Ji, L.L., Fogot, D.L. and Fretwell, V.S. (2007) Effect of exercise duration on postprandial hypertriglyceridemia in men with metabolic syndrome. Journal of Applied Physiology, 103, 1339-1345. doi:10.1152/japplphysiol.00181.2007

[8] Belli, T., et al. (2009) Impacto do exercício físico agudo no perfil metabólico pós prandial em adultos fisicamente aptos. Revista Brasileira de Cineantropometria e Desempenho Humano, 11, 314-319.

[9] Katsanos, C.S., Grandjean, P.W. and Moffat, R.J. (2004). Effects of low and moderate exercise intensity on postprandial lipemia and postheparin plasma lipoprotein li- pase activity in physically active men. Journal of Applied Physiology, 96, 181-188.

doi:10.1152/japplphysiol.00243.2003

[10] Ferreira, A.P. Ferreira, C.B., Souza, V.C., et al. (2011) The influence of intense intermittent versus moderate continuous exercise on postprandial lipemia. Clinics, 66, 535541. doi:10.1590/S1807-59322011000400003

[11] Pfeiffer, M., et al. (2005) The influence of walking performed immediately before meals with moderate fat content on post prandial lipemia. Lipids in Health and Disease, 4, 24. doi:10.1186/1476-511X-4-24

[12] Zhang, J.Q., Thomas, T.R. and Ball, S.D. (1998) Effect of exercise timing on postprandial lipemia and HDL cholesterol subfractions. Journal of Applied Physiology, 85, 1516-1522.

[13] Departamento De Aterosclerose Da Sociedade Brasileira De Cardiologia (2007) IV Diretriz Brasileira Sobre Dislipidemias e Prevenção da aterosclerose. Arquivos Brasileitos de Cardiologia, 88.

[14] Matsudo, S., Araújo, T., Marsudo, V., et al. (2001) Questinário internacional de atividade física (IPAQ): Estudo de validade e reprodutibilidade no Brasil. Revista Brasileira de Atividade Física e Saúde, 6, 05-18.

[15] Guedes, D.P., Lopes, C.C. and Guedes, J.E.R.P. (2005) Reprodutibilidade e validade do Questionário Internacional de Atividade Física em adolescentes. Revista Brasileira de Medicina do Esporte, 11.

[16] Paton, C.M., et al. (2006) Hemostatic response to postprandial lipemia before and after exercise training. Journal of Applied Physiology, 101, 316-321. doi:10.1152/japplphysiol.01363.2005

[17] Michelotto, M.A., et al. (2010) Relação de indicadores antropométricos com fatores de risco para doença cardiovascular. Arquivos Brasileiros de Cardiologia, 94, 478485. doi:10.1590/S0066-782X2010005000012

[18] Rondon, M.U.P.B., et al. (1998) Comparação entre a Prescrição de Intensidade de Treinamento Físico Baseada na Avaliação Ergométrica Convencional e na Ergoespirométric. Arquivos Brasileiros de Cardiologia, 70, 159166. doi:10.1590/S0066-782X1998000300004

[19] Caputo, F., Greco, C.C. and Denadai, B.S. (2005) Efeitos do estado e especificidade do treinamento aeróbio na relação $\% \mathrm{VO}_{2} \max$ versus $\% \mathrm{FCmax}$ durante o ciclismo. Arquivos Brasileiros de Cardiologia, 84, 20-23. doi:10.1590/S0066-782X2005000100005

[20] Katsanos, S.C. (2006) Prescribing aerobic exercise for the regulation of postprandial lipid metabolism. Sports Medicine, 36, 547-560. doi:10.2165/00007256-200636070-00001

[21] Petridou, A. et al. (2004) Effect of exercise performed immediately before a meal of moderate fat content on postprandial lipaemia. British Journal of Nutrition, 91, 683687. doi:10.1079/BJN20041097

[22] Blackburn, P., et al. (2003) Contribution of visceral adiposity to the exaggerated postprandial lipemia of Men with impaired Glucose Tolerance. Diabetes Care, 26, 3303-3309. doi:10.2337/diacare.26.12.3303 